\title{
Spinopelvic Alignment and Low Back Pain before and after Total Knee Arthroplasty
}

\author{
Atsushi Kitagawa, Junya Yamamoto, Mitsunori Toda, Yasushi Hashimoto \\ Department of Orthopaedic Surgery, Hyogo Rehabilitation Center Hospital, Kobe, Japan
}

\section{Study Design: Prospective cohort study.}

Purpose: This study aims to examine changes in spinopelvic alignment, sagittal global balance, and low back pain (LBP) following the removal of knee flexion contracture by total knee arthroplasty (TKA).

Overview of Literature: The limitation of the knee extension was correlated with the decrease in lumbar lordosis (LL). Currently, there are no studies evaluating the spinopelvic alignment and LBP before and after TKAs.

Methods: Sagittal spinopelvic alignment was evaluated in 110 subjects using radiographs of the whole spine. Parameters measured in this study included sagittal vertical axis (SVA), LL, sacral slope (SS), pelvic tilt (PT), and pelvic incidence (PI). The distribution of sagittal plane modifier grade was evaluated according to the Scoliosis Research Society-Schwab classification of adult spinal deformity (ASD). Consequently, personal history related to LBP was obtained, and the association of pre- and postoperative LBP and spinopelvic alignment was investigated.

Results: Preoperatively, $66 \%$ of all subjects showed LBP and mostly exhibited anteriorly shifted global imbalance associated with a decrease in $L L$ and knee flexion contractures, and the subject who had severe flexion contracture of the knee joint showed more forwardly shifted global balance with backward PT and decrease in LL. After TKAs, the knee flexion contractures were eliminated in most cases, and one-third of subjects experienced decrease in LBP. However, SVA increased more and associated with slight decrease of PT and increase of SS. No significant differences were confirmed between pre- and postoperative values of LL and PI. In addition, there were no significant differences in postoperative values of spinopelvic parameters between subjects with and without relieved LBP.

Conclusions: Although one-third of subjects experienced decrease of LBP after TKAs, the sagittal global imbalance was not restored through the removal of knee flexion contracture.

Keywords: Low back pain; Knee; Spine; Arthroplasty; Contracture

\section{Introduction}

Adult spinal deformity (ASD) has multiple etiologies. However, current studies addressing sagittal spinal align- ment were mainly focused on the alignment of abovehip structures [1-3], and only few reports considered below-hip structures. A previous report indicated that the presence of patellofemoral pain correlated with sacral

Received Dec 3, 2019; Revised Apr 12, 2020; Accepted Apr 17, 2020

Corresponding author: Atsushi Kitagawa

Department of Orthopaedic Surgery, Hyogo Rehabilitation Center Hospital, 1070 Akebono-cho, Nishi-ku, Kobe 651-2181, Japan

Tel: +81-78-927-2727, Fax:+81-78-925-9203,E-mail: a_kitagawa@hwc.or.jp 
inclination and knee flexion position and changes in lumbar kyphosis [4]. Furthermore, another study revealed that the limitation of knee extension was correlated with the decrease in lumbar lordosis (LL), and low back pain (LBP) may be due to degenerative changes in the knee joint; such phenomenon has been regarded as "kneespine syndrome." In the study, LL was significantly less in subjects with a knee extension limitation greater than $5^{\circ}$ [5]. Meanwhile, more recent study demonstrated that knee flexion contractures influence the three-dimensional trunk kinematics during relaxed standing or level walking which leads to spinal imbalance [6]. These study results could provide critical information that would contribute to the assessment of the change in spinal alignment and LBP following the elimination of knee contracture. For severe sagittal global imbalance, knee flexion is thought to be one of the main compensatory mechanisms; thus, we hypothesized that change of knee flexion contracture might affect global alignment and pathogenesis of LBP. The purpose of this study is to investigate changes in spinopelvic alignment, sagittal global balance, and lumbar symptom following the removal of knee flexion contracture by total knee arthroplasty (TKA).

\section{Materials and Methods}

\section{Study design}

Consecutive patients were recruited from individuals admitted to the authors' institution for primary TKA. Among candidates, subjects who suffered from advanced osteoarthritis (OA) or rheumatoid arthritis (RA) associated with knee flexion contracture were enrolled the study. A total of 110 individuals (102 OA and eight RA) referred for TKA were enrolled in the study, of which 17 are males and 93 are females. The mean age at surgery was 75 years old (range, 52-91 years old). Body mass index was $25.7 \pm 3.7 \mathrm{~kg} / \mathrm{m}^{2}$. All subjects were classified as Kellgren-Lawrence grade 3 or 4 . All surgeries were performed under consistent and reproducible techniques for balancing flexion and extension gap and tension of collateral ligaments, using the same prosthesis design (Triathlon, Stryker, NJ, USA). The prevalence of LBP was assessed using the Japanese version of the Roland-Morris Questionnaire (RMQ), a self-rated health status measure. It was designed for patients to assess pain-related physical disability due to LBP. RMQ scores range from 0 (no disability) to 24 (maximum disability). Consequently, personal history related to LBP and knee pain was obtained before TKA and 1 year after TKA as well as data from the questionnaire regarding change of LBP between pre- and postoperation and whether the knee pain was caused after the appearance of LBP or vice versa. The exclusion criteria of the present study were as follows: prior spinal surgery, Cobb angle more than $20^{\circ}$, C7 Frontal imbalance larger than $5 \mathrm{~cm}$, and flexion contracture of the hip or the other side of the knee joint. The study design was approved by the relevant institutional ethics committee (2016-1602), and all participants provided informed consents.

\section{Radiographical analysis}

Standing lateral full-length radiographs of the whole spine were taken before surgeries. The neutral standing position was taken with the knee maximally extended being their tallest posture of hip and spine. The fingers were held on the clavicle with upper arms positioned approximately $45^{\circ}$ to the vertical [4]. After 1 month of intensive rehabilitation, radiographs of the entire spine and lateral knee with standing position were taken. A digital analysis of all radiographic measurements was done using a specific software program (SYNAPSE OP-A; Fuji Film, Tokyo, Japan). The parameters that measured in this study concerning sagittal spinopelvic alignment were sagittal vertical axis (SVA), shift of the sagittal C7 plumb line, LL, sacral slope (SS), pelvic tilt (PT), and pelvic incidence (PI). PI is calculated in the following equation: $\mathrm{PI}=\mathrm{PT}+\mathrm{SS}$. Moreover, based on these measurements, the distribution of sagittal plane modifier grade was evaluated according to the Scoliosis Research Society (SRS)-Schwab classification of ASD as follows [7]: (1) patient with a PT less than $20^{\circ}$ was classified with a PT modifier 0; (2) patient with a PT between $20^{\circ}$ and $30^{\circ}$ was classified with a PT modifier +; (3) patient with a PT value of greater than $30^{\circ}$ was classified with a PT modifier ++; (4) patient with a SVA of less than $40 \mathrm{~mm}$ was classified with an SVA modifier 0; (5) patient with a SVA between $40 \mathrm{~mm}$ and $95 \mathrm{~mm}$ was classified with an SVA modifier +; (6) patient with a SVA of greater than $95 \mathrm{~mm}$ was classified with an SVA modifier ++; (7) patient with a PI-LL value of less than $10^{\circ}$ was classified with a PI-LL modifier 0; (8) patient with a PI-LL value of between $10^{\circ}$ and $20^{\circ}$ was classified with a PI-LL modifier + ; and (9) patient with a PI-LL value of greater than $20^{\circ}$ was classified with a PI-LL modifier ++. 


\section{Statistical analysis}

For each parameter, differences between pre- and postoperative values were evaluated using the paired Student $t$-test. Pearson's correlation coefficient was conducted to analyze the relationship between maximum knee extension angle and each parameter. All $p<0.05$ were considered significant. All statistical analyses were performed using SPSS II ver. 11.0 (SPSS Inc., Chicago, IL, USA).

\section{Results}

\section{Sagittal balance before total knee arthroplasties}

Before TKAs, most of our subjects exhibited flexion contracture of the knee joint, and the average maximum knee extension angle was $-11.8^{\circ} \pm 10.1^{\circ}$. Preoperatively, C7 plumb line was located on an average of $55.5 \mathrm{~mm}$ anteriorly from the most posterior point of the sacral endplate. The mean value and the standard deviation of LL was $38.7^{\circ} \pm 17.0^{\circ}$, and values of each pelvic parameter (SS, PT, and PI) were $28.7^{\circ} \pm 10.1^{\circ}, 23.6^{\circ} \pm 9.5^{\circ}$, and $52.3^{\circ} \pm 10.7^{\circ}$, respectively (Table 1 ). In addition, the maximum knee extension angle demonstrated weak negative correlation with SVA and positive correlation with SS and LL, as illustrated in Table 2.

\section{Comparison of sagittal balance after TKAs and distribu- tion of sagittal plane modifier grade based on the SRS- Schwab classification system}

Postoperatively, the average maximum knee extension angle was $-3.2^{\circ} \pm 6.9^{\circ}(p<0.001)$, which indicated an improvement in knee flexion contractures in most cases. The mean value and the standard deviation of SVA was $60.9 \pm 39.8 \mathrm{~mm}$, and significant differences were noted between pre- and postoperative values. C7 plumb line was shifted more anteriorly at an average of $5.6 \mathrm{~mm}$. For other spinopelvic parameters, there were no significant differences observed between pre- and postoperative values of LL $(39.6 \pm 17.5)$ and PI $\left(52.2^{\circ} \pm 17.5^{\circ}\right)$. However, slight decrease and increase in values of PT $\left(22.3^{\circ} \pm 9.0^{\circ}\right)$ and SS $\left(29.8^{\circ} \pm 10.7^{\circ}\right)$ were confirmed postoperatively (Table 1$)$. Despite the small maximum knee extension, negative correlation was still confirmed between the maximum knee extension angle and SVA (Table 2). The distribution of pre- and postoperative modifier grade for all subjects was summarized in Fig. 1. Of 110 subjects, numbers and proportions of subjects with a normal modifier grade were 40 (36.3\%) in PT, 40 (36.3\%) in SVA, and 50 (45.5\%) in PILL, respectively, before TKAs. Postoperatively, these numbers and proportions were 48 (43.6\%) in PT, 35 (31.8\%) in SVA, and 49 (44.5\%) in PI-LL. In addition, preoperative numbers and proportions of subjects with poorer modi-

Table 1. Comparison of the sagittal spinopelvic alignment parameters and the maximum knee extension angle before and after total knee arthroplasties

\begin{tabular}{lccc} 
Variable & Preoperation & Postoperation & $p$-value \\
\hline Sagittal vertical axis $(\mathrm{mm})$ & $55.5 \pm 42.2$ & $60.9 \pm 39.8$ & 0.029 \\
\hline Lumber lordosis $\left({ }^{\circ}\right)$ & $38.7 \pm 17.0$ & $39.6 \pm 17.5$ & 0.147 \\
\hline Sacral slope $\left(^{\circ}\right)$ & $28.7 \pm 10.1$ & $29.8 \pm 10.7$ & 0.049 \\
\hline Pelvic tilt $\left(^{\circ}\right)$ & $23.6 \pm 9.50$ & $22.3 \pm 9.0$ & $<0.001$ \\
\hline Pelvic incidence $\left({ }^{\circ}\right)$ & $52.3 \pm 10.7$ & $52.2 \pm 11.6$ & 0.870 \\
\hline Knee extension angle $\left({ }^{\circ}\right)$ & $-11.8 \pm 10.1$ & $-3.2 \pm 6.9$ & $<0.001$ \\
\hline
\end{tabular}

Values are presented as mean \pm standard deviation.

Table 2. Correlation between the maximum knee extension angle and the values of spinpelvic parameters

\begin{tabular}{lcccc} 
Knee extension angle & Sagittal vertical axis & Sacral slope & Pelvic tilt & Pelvic incidence \\
Pre-TKA $\left(^{\circ}\right)$ & $-0.279^{*}$ & $0.202^{*}$ & -0.038 & 0.158 \\
Post-TKA $\left(^{\circ}\right)$ & $-0.319^{* *}$ & -0.017 & 0.069 & 0.040 \\
\hline
\end{tabular}

TKA, total knee arthroplasty.

${ }^{*} p<0.05,{ }^{* *} p<0.01$; by Pearson's correlation coeficient. 


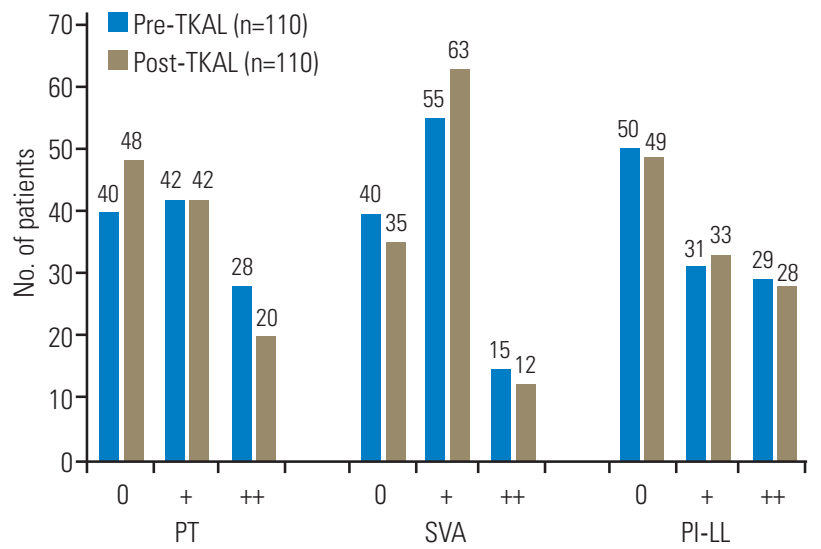

Fig. 1. Distribution of patients according to the severity of the sagittal plane modifier grades between pre- and post-TKAs. TKA, total knee arthroplasty; PT, pelvic tilt; SVA, sagittal vertical axis; PI, pelvic incidence; LL, lumber lordosis.

Table 3. Comparison of demographics and the sagittal spinopelvic alignment parameters between patients with and without LBP before total knee arthroplasties

\begin{tabular}{lccc} 
Variable & With LBP & Without LBP & $p$-value \\
\hline Total no. of subjects & 73 & 37 & \\
\hline Mean age $(\mathrm{yr})$ & $74.4 \pm 7.0$ & $76.0 \pm 6.8$ & 0.260 \\
\hline Body mass index $\left(\mathrm{kg} / \mathrm{m}^{2}\right)$ & $25.7 \pm 3.7$ & $24.9 \pm 3.7$ & 0.280 \\
\hline Knee extension angle $\left(^{\circ}\right)$ & $-11.1 \pm 10.9$ & $-12.9 \pm 8.2$ & 0.340 \\
\hline Sagittal vertical axis $(\mathrm{mm})$ & $61.7 \pm 43.2$ & $42.8 \pm 38.1$ & 0.024 \\
\hline Sacral slope $\left(^{\circ}\right)$ & $28.2 \pm 11.0$ & $29.6 \pm 8.1$ & 0.454 \\
\hline Pelvic tilt $\left({ }^{\circ}\right)$ & $24.6 \pm 10.0$ & $21.6 \pm 8.1$ & 0.099 \\
\hline Pelvic incidence $\left(^{\circ}\right)$ & $52.8 \pm 11.1$ & $52.8 \pm 11.1$ & 0.470 \\
\hline Lumber lordosis $\left({ }^{\circ}\right)$ & $36.6 \pm 19.4$ & $42.8 \pm 10.0$ & 0.030 \\
\hline
\end{tabular}

Values are presented as mean \pm standard deviation.

LBP, low back pain.

fier grade $(+,++)$ were $70(63.7 \%)$ in PT, $70(63.7 \%)$ in SVA, and 60 (54.5\%) in PI-LL, respectively. In contrast, postoperative numbers and proportions were 62 (56.4\%), 75 (68.2\%), and 61 (55.5\%), respectively. These results indicated no significant differences between numbers and proportions of pre- and postoperative sagittal plane modifier grade.

\section{Comparison of subjects with and without low back pain before total knee arthroplasties}

Seventy-three of 110 subjects (66.4\%) showed LBP associated with knee pain, preoperatively. In the group of subjects with LBP, the mean RMQ score was 8.5 \pm 5.7 . Demographics and the maximum knee extension angles

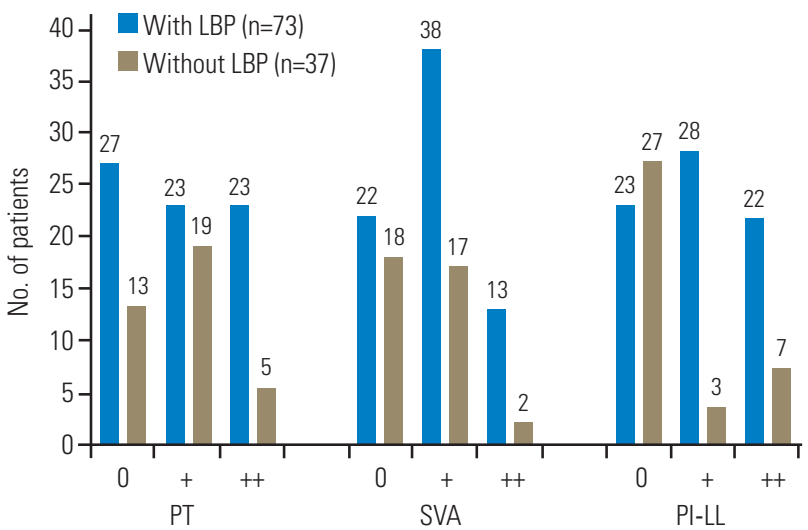

Fig. 2. Distribution of patients according to the severity of the sagittal plane modifier grades between patients with and without LBP before total knee arthroplasties. LBP, low back pain; PT, pelvic tilt; SVA, sagittal vertical axis; PI, pelvic incidence; LL, lumber lordosis.

were not significantly different between subjects who experienced LBP and did not experience LBP. However, subjects with LBP showed significantly larger SVA and smaller LL compared with values of subjects without LBP (Table 3). The distribution of each modifier grade for all subjects who experienced or did not experience LBP preoperatively was summarized in Fig. 2. In 73 subjects with LBP, numbers and proportions of subjects with a normal modifier grade were 27 (37.0\%) in PT, 22 (30.1\%) in SVA, and 23 (31.5\%) in PI-LL, respectively. In contrast, numbers and proportions were 13 (35.1\%), 18 (48.6\%), and 27 (73.0\%), respectively, in 37 subjects who did not experience LBP before TKAs. Moreover, in the group with LBP, numbers and proportions of subjects with poorer modifier grade $(+,++)$ were $46(63.0 \%)$ in PT, 51 (69.9\%) in SVA, and 50 (68.5\%) in PI-LL, respectively. In contrast, numbers and proportions were 24 (64.9\%), 19 (51.4\%), and $10(27.0 \%)$, respectively, in the group without LBP. These results indicated that proportion of normal grades of SVA and PI-LL were smaller and poorer grades were larger in the group of subjects who experienced LBP than the group who did not experience LBP.

\section{Comparison of low back pain before and after total knee arthroplasties}

Fifty-three of 110 subjects (48.2\%) still exhibited LBP although their knee pain was relieved postoperatively, and the mean RMQ score was $6.2 \pm 5.1$ in the group of subjects with LBP. In the group of subjects who showed LBP preoperatively, 25 of 73 subjects (34.2\%) experienced 

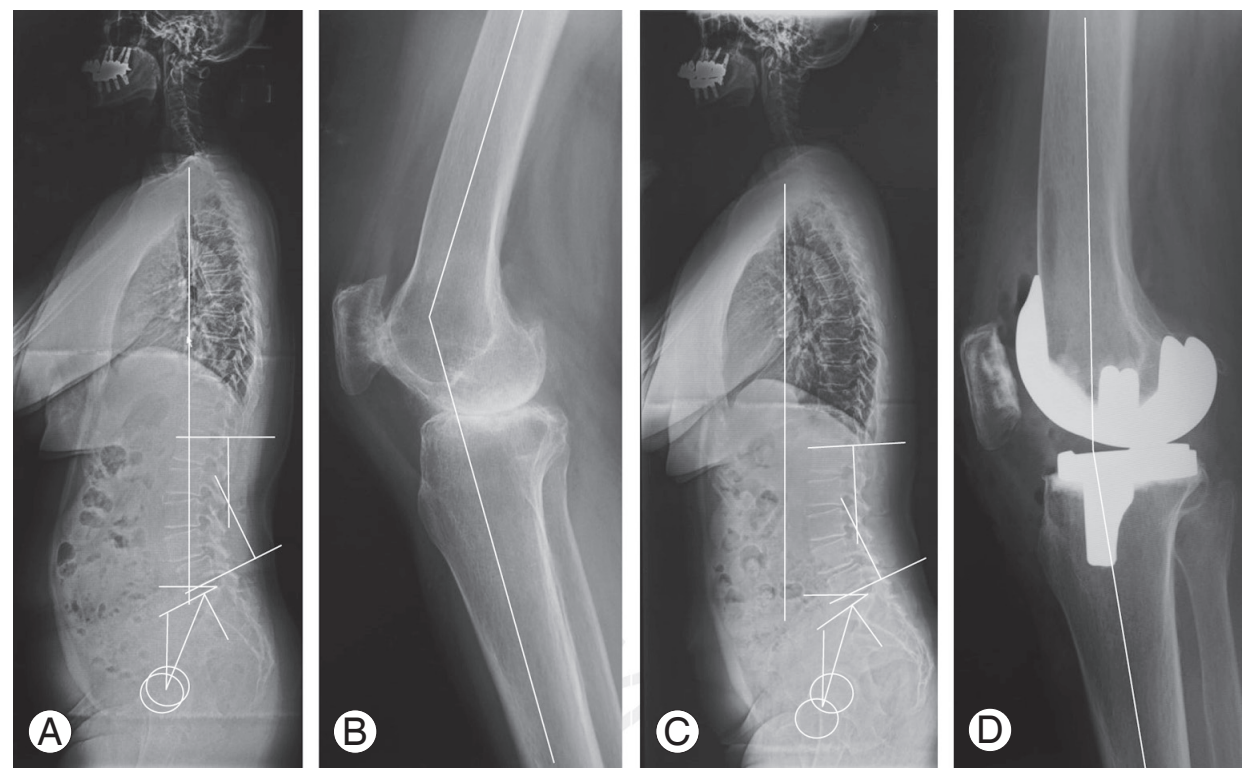

Fig. 3. Case example of a 60 -year-old woman. (A) A preoperative radiography showing the whole spine standing lateral which exhibited the following findings: SVA, $35 \mathrm{~mm} ; \mathrm{LL}, 27^{\circ}, \mathrm{SS}, 28^{\circ}, \mathrm{PT}, 22^{\circ}, \mathrm{PI}, 50^{\circ}$. (B) A preoperative lateral knee radiography which indicated $32^{\circ}$ of flexion contracture. (C) A postoperative whole spine standing lateral radiography showing more anterior-shifted global balance with the following findings: SVA, $85 \mathrm{~mm} ; \mathrm{LL}, 24^{\circ}, \mathrm{SS}, 35^{\circ} ; \mathrm{PT}, 15^{\circ} ; \mathrm{Pl}, 5^{\circ}$. (D) A postoperative lateral knee radiography indicating $24^{\circ}$ of decreased flexion contracture. SVA, sagittal vertical axis; LL, lumber lordosis; SS, sacral slope; PT, pelvic tilt; PI, pelvic incidence.

Table 4. Comparison of demographics and the sagittal spinopelvic alignment parameters between patients with reduced and not reduced LBP after total knee arthroplasties

\begin{tabular}{lccc} 
Variable & $\begin{array}{c}\text { Reduced } \\
\text { LBP }\end{array}$ & $\begin{array}{c}\text { Not reduced } \\
\text { LBP }\end{array}$ & $p$-value \\
\hline Total no. of subjects & 25 & 43 & \\
\hline Mean age $(\mathrm{yr})$ & $74.0 \pm 7.0$ & $74.6 \pm 7.0$ & 0.250 \\
\hline Body mass index $\left(\mathrm{kg} / \mathrm{m}^{2}\right)$ & $25.5 \pm 3.8$ & $25.9 \pm 3$. & 0.260 \\
\hline Knee extension angle $\left({ }^{\circ}\right)$ & $-3.1 \pm 7.8$ & $-3.2 \pm 4.8$ & 0.310 \\
\hline Sagittal vertical axis $(\mathrm{mm})$ & $54.2 \pm 38.0$ & $62.2 \pm 37.2$ & 0.032 \\
\hline Sacral slope $\left({ }^{\circ}\right)$ & $29.3 \pm 10.4$ & $30.0 \pm 9.1$ & 0.462 \\
\hline Pelvic tilt $\left({ }^{\circ}\right)$ & $23.2 \pm 9.6$ & $21.4 \pm 8.7$ & 0.120 \\
\hline Pelvic incidence $\left({ }^{\circ}\right)$ & $52.2 \pm 11.2$ & $52.2 \pm 11.0$ & 0.482 \\
\hline Lumber lordosis $\left({ }^{\circ}\right)$ & $38.2 \pm 18.8$ & $40.0 \pm 17.0$ & 0.102 \\
\hline
\end{tabular}

Values are presented as mean \pm standard deviation.

LBP, Iow back pain.

a decrease or disappearance of LBP, and the rest of the 48 subjects manifested no improvement of LBP after TKAs. No subjects reported LBP postoperatively in the group of subjects who did not show LBP preoperatively. Although there were no significant differences in postoperative values in demographics and maximum knee extension angles, there was a marked difference in the value of SVA between subjects who experienced and did not experience decrease of LBP (Table 4). In the group of the subjects who experienced LBP preoperatively, 43 of 73 subjects (59\%) had experienced LBP prior to the manifestation of knee pain, and 24 subjects (33\%) had experienced knee pain prior to appearance of LBP. The rest of the six subjects were not able to indicate the primary reason of pain. Postoperatively, in the group of subjects who experienced reduced LBP, 16 of 25 subjects (64\%) had experienced knee pain before the occurrence of LBP, and seven of 25 subjects (28\%) had experienced LBP before the manifestation of knee pain. However, primary lesions of pain were not confirmed for the rest of the two subjects.

\section{Discussion}

To the best of our knowledge, this is the first study demonstrating the relationship of sagittal global alignment and LBP before and after TKAs, and the most critical finding of the present study is that the anteriorly shifted sagittal global imbalance was not compensated by the removal of knee flexion contracture in a short postoperative period. Normal sagittal alignment of the global spine is essential in keeping the gravity line centered in the 
pelvis and maintaining the standing position with little muscle effort. Once the sagittal alignment is abnormal, more energy is required for the body to sustain its balance without external support [8-10]. To maintain the sagittal balance of the spine, compensatory mechanisms are needed in the spine, the pelvis, and/or the lower limb areas [9]. In clinical practice, global balance is typically determined by SVA, and the loss of sagittal balance is significant if the $\mathrm{C} 7$ plumb line is $\geq 5 \mathrm{~cm}$ either anterior or posterior to the sacral promontory. Preoperatively, our result on the $\mathrm{C} 7$ plumb line indicated that candidates for TKA mostly exhibited anteriorly shifted global imbalance associated with knee flexion contracture, which suggested insufficient compensatory mechanisms in the local segment. For each spinopelvic parameter, the normal values have been described by several authors in their respective population, and the effect of ethnicity on skeletal growth have also been demonstrated by previous reports $[11,12]$. Recent reports indicated that the mean value of $L L$ is $51^{\circ}$, ranging from $29^{\circ}$ to $76^{\circ}$ from L1 to S1, based on a large cohort study from our country [13]. Compared with the study result, the mean value of our subjects $\left(38.7^{\circ} \pm 17.0^{\circ}\right)$ was relatively small within normal range, which implied that one of the compensatory mechanism of the local segment, lumbar hyperlordosis, did not function well. On the other hand, PI was proposed to determine the correlation between sagittal pelvic orientation and the extent of lordosis. The value of PI varies between individuals but not with positioning in an individual, and a higher value is associated with more LL, and lower value, in constant, is associated with less lordosis.

Considering the result of our values of LL, the value of PI was expected to be lower than normal. However, our result of PI $\left(52.3^{\circ} \pm 10.7^{\circ}\right)$ was within normal range, compared with data from previous studies, in which the mean value was 54 with a range from 43 to 62 [13]. Therefore, intrinsic morphology of the pelvis was relatively normal, and the PI-LL mismatch, confirmed in more than 50\% of all subjects, might be mainly influenced by low value of LL in our subjects. The cohort study also reported that the mean value of PT was about 18 , ranging from 10 to 43 , and the mean value of SS was 36, ranging from 10 to 45 [13]. Compared with these results, the mean values of our subjects (PT, $23.6^{\circ} \pm 9.50^{\circ}$; SS, $28.7^{\circ} \pm 10.1^{\circ}$ ) indicated relatively high $\mathrm{PT}$ and low SS, which equates to a slight retroversion of the pelvis and suggested that one of the compensatory mechanism of the local segment, pelvic retroversion, could function but is not enough to compensate global imbalance.

Other reports from our country indicated that LL and SS decreased significantly by more than $5^{\circ}$ in subjects with knee flexion contractures which suggested that the knee and spine affect each other [14]. Considering that our subjects who had severe flexion contracture of the knee joint showed more forwardly shifted global balance associated with backward PT and decrease of LL, which is consistent with the previous study, forwardly shifted global imbalance could be possibly influenced by inflexibility of knee joints.

Our postoperative alignment results indicated a successful elimination of the flexion contracture in most subjects. In non-disease population, simulated knee flexion resulted in a decreased femoropelvic angle and LL and the anterior shift of sagittal balance without altering the pelvic position [15]. Although the femoropelvic angle was not evaluated in the study, no flexion contracture of hip was confirmed in our subject; thus, we anticipated the posterior shift of the C7 plumb line and increase of LL by improvement of the knee flexion contracture after TKAs. However, anteriorly shifted imbalance was not compensated in most cases, while the center of the gravity line in the standing position shifted more anteriorly without counterbalanced by the lordotic lumbar spine. The representative case is shown in Fig. 3. Previous reports indicated that a change in the flexion contracture after TKA affects the SS only in a small number of subjects group, in which value of PT was not changed. Therefore, pre- and post operative values of PI were inconsistent by unknown reason [16]. In our study, the number of subjects was greater, and the result of pre- and postoperative values of PI were consistent. Therefore, slight decrease and increase in postoperative values of PT and SS might be caused by a slight anteversion of backward tilted pelvis without altering the intrinsic pelvic morphology. These observation, in contrast to our hypothesis, suggested that the sagittal global imbalance may not be restored after the removal of knee flexion contracture by TKA in a short postoperative period.

Traditionally, LBP is considered to be nonspecific, and the etiology of $80 \%$ to $90 \%$ of LBP cases have thought to be unknown for decades. However, LBP symptoms might be derived from many potential anatomic sources, such as nerve roots, muscle, fascial structures, bones, joints, and intervertebral discs [17]. Sixty-six percent of our sub- 
jects experienced LBP associated with knee degenerative changes, which is a higher prevalence than that reported in the general population [18], and the result of the cohort study indicated that 3.9\% experienced chronic LBP in ages from 20 to 79 years old in our country [19]. Although the prevalence of knee pain in the Japanese elderly cohorts is about 33\% [20], to our knowledge, there have been no epidemiological study regarding the relationship between LBP and knee pain. The Japanese version of the RMQ score is a useful scale which is easy to use, with proven reliability, validity, and responsiveness when assessing patients with LBP [21]. Our results suggested that about two-thirds of TKA candidates experienced disability due to LBP preoperatively. Although evaluation of pain generator requires thorough investigation of many factors and we only examined functional status of knee joints, our results suggested a causal relationship between LBP and degeneration of the knee joint. The sagittal modifiers of the SRS-Schwab classification of ASD is useful and a practical tool detecting various grades of deformity [22], which have been proven to correlate with health-related quality of life [23-25]. In general, the mean SVA shifts forward in the aging population, and a strong correlation exists between a positive shift in the SVA and back pain and fatigue. Our study subjects were mostly elderly, and the result of modifier grades indicated that more than $60 \%$ of subjects with LBP showed poor grades in each of three parameters. The result of spinopelvic alignment parameters also suggested a correlation between LBP and the specific pattern, presenting as large SVA and small LL. Although no correlation between LBP and knee flexion contracture was confirmed, high involvement of the LBP in TKA candidates implied the association between ASD and knee degeneration.

Despite the decompensated sagittal imbalance after TKAs, about one-third of subjects experienced a decrease in LBP, and a slight improvement of the RMQ score was confirmed. However, no distinction was confirmed in the postoperative maximum knee extension angle and alignment parameters except for SVA between subjects with and without relieved LBP. Speculating factors related to change of LBP after TKAs could provide critical information in revealing whether the knee flexion contracture results in a decrease of LL and causes LBP or vice versa. Considering the result of the questionnaire regarding the primary lesion of pain, LBP tended to decrease in subjects who have experienced LBP secondary to knee pain. Therefore, if subjects have knee degeneration first, LBP could be improved by removal of the knee flexion contracture associated with slight posterior shift of the C7 plumb line.

There were several limitations of the present study. First, the number of our subjects was relatively small, thus, reducing the statistical power of the study. Second, the period of radiographical analysis was short, and the subjects were evaluated retrospectively with a follow-up period of only 1 year. Third, most of our subjects are the elderly, female, and had a relatively high BMI. Fourth, we only investigated the alignment parameters and LBP which could be influenced by multiple factor not considered in the study. Therefore, further studies with more subjects, longer follow-up period, and wider range of age and BMI values may be necessary to clarify the involvement of degenerative knee changes in development of LBP, which would provide beneficial information for TKA candidates.

\section{Conclusions}

Sixty-six percent of all subjects showed LBP before TKAs and exhibited forwardly inclined global alignment associated with small LL and backward PT. Although one-third of subjects showed relief of LBP after TKAs, $43 \%$ of all subjects still experienced LBP, and the sagittal imbalance was not restored following the removal of knee flexion contracture by TKA during a short postoperative period.

\section{Conflict of Interest}

No potential conflict of interest relevant to this article was reported.

\section{References}

1. Offierski CM, MacNab I. Hip-spine syndrome. Spine (Phila Pa 1976) 1983;8:316-21.

2. Takemitsu Y, Harada Y, Iwahara T, Miyamoto M, Miyatake Y. Lumbar degenerative kyphosis: clinical, radiological and epidemiological studies. Spine (Phila Pa 1976) 1988;13:1317-26.

3. Yoshimoto H, Sato S, Masuda T, et al. Spinopelvic alignment in patients with osteoarthrosis of the hip: a radiographic comparison to patients with low back pain. Spine (Phila Pa 1976) 2005;30:1650-7.

4. Tsuji T, Matsuyama Y, Goto M, et al. Knee-spine 
syndrome: correlation between sacral inclination and patellofemoral joint pain. J Orthop Sci 2002;7:51923.

5. Murata Y, Takahashi K, Yamagata M, Hanaoka E, Moriya $\mathrm{H}$. The knee-spine syndrome: association between lumbar lordosis and extension of the knee. J Bone Joint Surg Br 2003;85:95-9.

6. Harato K, Nagura T, Matsumoto H, Otani T, Toyama Y, Suda Y. A gait analysis of simulated knee flexion contracture to elucidate knee-spine syndrome. Gait Posture 2008;28:687-92.

7. Schwab F, Ungar B, Blondel B, et al. Scoliosis Research Society-Schwab adult spinal deformity classification: a validation study. Spine (Phila Pa 1976) 2012;37:1077-82.

8. Obeid I, Berjano P, Lamartina C, Chopin D, Boissiere L, Bourghli A. Classification of coronal imbalance in adult scoliosis and spine deformity: a treatmentoriented guideline. Eur Spine J 2019;28:94-113.

9. Barrey C, Roussouly P, Le Huec JC, D’Acunzi G, Perrin G. Compensatory mechanisms contributing to keep the sagittal balance of the spine. Eur Spine J 2013;22 Suppl 6(Suppl 6):S834-41.

10. Obeid I, Hauger O, Aunoble S, Bourghli A, Pellet N, Vital JM. Global analysis of sagittal spinal alignment in major deformities: correlation between lack of lumbar lordosis and flexion of the knee. Eur Spine J 2011;20(Suppl 5):681-5.

11. Gilsanz V, Skaggs DL, Kovanlikaya A, et al. Differential effect of race on the axial and appendicular skeletons of children. J Clin Endocrinol Metab 1998;83:1420-7.

12. Lonner BS, Auerbach JD, Sponseller P, Rajadhyaksha AD, Newton PO. Variations in pelvic and other sagittal spinal parameters as a function of race in adolescent idiopathic scoliosis. Spine (Phila Pa 1976) 2010;35:E374-7.

13. Asai Y, Tsutsui S, Oka H, et al. Sagittal spino-pelvic alignment in adults: the Wakayama Spine Study. PLoS One 2017;12:e0178697.

14. Yanagisawa S, Sato N, Shimizu M, Saito K, Yamamoto A, Takagishi K. Relation among the knee, sagittal spinal alignment, and the spinal range of motion: investigation in local medical check-ups using the SpinalMouse. Asia Pac J Sports Med Arthrosc Rehabil Technol 2015;2:68-71.
15. Lee CS, Park SJ, Chung SS, Lee KH. The effect of simulated knee flexion on sagittal spinal alignment: novel interpretation of spinopelvic alignment. Eur Spine J 2013;22:1059-65.

16. Lee SM, Yoon MG, Moon MS, Lee BJ, Lee SR, Seo YH. Effect of correction of the contractured flexed osteoarthritic knee on the sagittal alignment by total replacement. Asian Spine J 2013;7:204-11.

17. Allegri M, Montella S, Salici F, et al. Mechanisms of low back pain: a guide for diagnosis and therapy: version 2. F1000Res 2016;5:F1000 Faculty Rev-1530.

18. Meucci RD, Fassa AG, Faria NM. Prevalence of chronic low back pain: systematic review. Rev Saude Publica 2015;49:1.

19. Fujii T, Matsudaira K, Oka H. The association between compensation and chronic disabling back pain. J Orthop Sci 2012;17:694-8.

20. Muraki S, Oka H, Akune T, et al. Prevalence of radiographic knee osteoarthritis and its association with knee pain in the elderly of Japanese population-based cohorts: the ROAD study. Osteoarthritis Cartilage 2009; 17:1137-43.

21. Kyrola K, Repo J, Mecklin JP, Ylinen J, Kautiainen $\mathrm{H}$, Hakkinen A. Spinopelvic changes based on the simplified SRS-Schwab adult spinal deformity classification: relationships with disability and healthrelated quality of life in adult patients with prolonged degenerative spinal disorders. Spine (Phila Pa 1976) 2018;43:497-502.

22. Suzukamo Y, Fukuhara S, Kikuchi S, et al. Validation of the Japanese version of the Roland-Morris Disability Questionnaire. J Orthop Sci 2003;8:543-8.

23. Glassman SD, Bridwell K, Dimar JR, Horton W, Berven S, Schwab F. The impact of positive sagittal balance in adult spinal deformity. Spine (Phila Pa 1976) 2005;30:2024-9.

24. Lafage V, Schwab F, Patel A, Hawkinson N, Farcy JP. Pelvic tilt and truncal inclination: two key radiographic parameters in the setting of adults with spinal deformity. Spine (Phila Pa 1976) 2009;34:E599606.

25. Schwab FJ, Blondel B, Bess S, et al. Radiographical spinopelvic parameters and disability in the setting of adult spinal deformity: a prospective multicenter analysis. Spine (Phila Pa 1976) 2013;38:E803-12. 